\title{
Efficient Seventh-Order Hybrid Block Process for Solving Stiff Second Order Ordinary Differential Equations
}

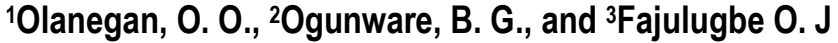 \\ 1 Statistics Department, Federal Ile-Oluji, Ondo State, Nigeria \\ 2Mathematics Department, Federal University, Oye Ekiti, Ekiti State, Nigeria \\ ${ }^{3}$ Department of Basic Medical Science, College of Health Sciences, ljero Ekiti, Ekiti State, Nigeria \\ 1Phone No: +2347035483705 \\ E-mail: olaolanegan@fedpolel.edu.ng
}

\begin{abstract}
In this research, we proposed a derivation and application of an accurate hybrid block method for the direct integration of second order ordinary differential equations with initial value problems. The method developed by way of collocating and interpolating the power series approximation to give a continuous linear multistep method. The evaluation of the continuous method at the grid and off grid points produced the discrete block method. The basic properties such as order, error constant, zero stability, consistency and convergence of the method were properly examined. The block method derived produces more efficient results when compared with the work carried out by some authors to solve stiff second order ordinary differential equations.
\end{abstract}

Keywords: Power Series, Collocation and Interpolation, Block Method, Initial Value Problems, and Stiff ODEs

Proceedings Reference Format

Olanegan, O. O., Ogunware, B. G. \& Fajulugbe O. J. (2021): Efficient Seventh-Order Hybrid Block Process for Solving Stiff Second Order Ordinary Differential Equationsl. Proceedings of the 27th iSTEAMS Multidisciplinary Innovations \& Technology Transfer (MINTT) Conference. Academic City University College, Accra, Ghana. June, 2021. Pp 399-406 www.isteams.net/ghana2021.

DOI - https://doi.org/ 10.22624/AIMS/iSTEAMS-2021/N27P37

\section{INTRODUCTION}

This work focus on the second order ordinary differential equations of the form:

$y^{\prime \prime}=f\left(x, y, y^{\prime}\right), y(a)=y_{0}, y^{\prime}(a)=\beta$

(1)

With initial conditions $y(a)=y_{0}, y^{\prime}(a)=\beta$ which has been extensively discussed by several scholars using different approach such as reduction process, predictor-corrector procedure, and Taylor series approach to proffer solutions to equation. Some of these researchers are Olanegan et al (2018), Waeleh (2017), Adoghe (2017), Abada (2017), Olanegan et al., (2015), Awoyemi et al (2015). Some noticeable drawbacks were observed in the aforementioned approaches like complicated programming procedure of writing subroutines, low order of accuracy, and more effort in developing another scheme to be of the same order with the predictor. Hence, Authors, like Jator (2007), Awoyemi (2001), Omar and Kuboye (2016), Ogunware and Omole (2020), Akeremale et al., (2020), developed Block methods for solving second order ODEs with IVPs directly to overcome these challenges to bring improvement in numerical analysis. 
The accuracy of the block methods were far better than the other previous methods. The uniqueness of block method is that in each application, the solution will be obtained concurrently at various points. It is in the light of this that we focus this research on the development of hybrid block method for the direct numerical solution of second order ODEs with IVPs.

\section{DERIVATION OF THE METHOD}

Consider a power series of the form (2) as an approximate solution to equation

$y(x)=\sum_{j=0}^{(\phi+\theta)} a_{j} x^{j}$

where $\phi$ and $\theta$ are the number of the interpolation and collocation points respectively.

The second derivative of (2) is obtained as

$y^{\prime \prime}=\sum_{j=2}^{(\phi+\theta)} j(j-1) a_{j} x^{j-2}$

combining equations (1) and (3) yields the differential system of the form

$y^{\prime \prime}=\sum_{j=0}^{(\phi+\theta)} j(j-1) a_{j} x^{j-2}=f\left(x, y, y^{\prime}\right)$

Collocating (4) at $x=x_{n+j}, j=0, \frac{1}{8}, \frac{1}{4}, \frac{3}{8}, \frac{1}{2}, \frac{5}{8}$ and interpolating (2) at $x_{x=x_{n+j}}, j=\frac{1}{8}, \frac{1}{4}$ gives arrangement of non-linear system in equation (5)

$\left[\begin{array}{cccccccc}1 & x_{n+1 / 8} & x_{n+1 / 8}^{2} & x_{n+1 / 8}^{3} & x_{n+1 / 8}^{4} & x_{n+1 / 8}^{5} & x_{n+1 / 8}^{6} & x_{n+1 / 8}^{7} \\ 1 & x_{n+1 / 4} & x_{n+1 / 4}^{2} & x_{n+1 / 4}^{3} & x_{n+1 / 4}^{4} & x_{n+1 / 4}^{5} & x_{n+1 / 4}^{6} & x_{n+1 / 4}^{7} \\ 0 & 0 & 2 & 6 x_{n} & 24 x_{n}^{2} & 120 x_{n}^{3} & 720 x_{n}^{4} & 5040 x_{n}^{5} \\ 0 & 0 & 2 & 6 x_{n+1 / 8} & 24 x_{n+1 / 8}^{2} & 120 x_{n+1 / 8}^{3} & 720 x_{n+1 / 8}^{4} & 5040 x_{n+1 / 8}^{5} \\ 0 & 0 & 2 & 6 x_{n+1 / 4} & 24 x_{n+1 / 4}^{2} & 120 x_{n+1 / 4}^{3} & 720 x_{n+1 / 4}^{4} & 5040 x_{n+1 / 4}^{5} \\ 0 & 0 & 2 & 6 x_{n+3 / 8} & 24 x_{n+3 / 8}^{2} & 120 x_{n+3}^{3} & 720 x_{n+3 / 8}^{4} & 5040 x_{n+3 / 8}^{5} \\ 0 & 0 & 2 & 6 x_{n+1 / 2} & 24 x_{n+1 / 2}^{2} & 120 x_{n+1 / 2}^{3} & 720 x_{n+1 / 2}^{4} & 5040 x_{n+1 / 2}^{5} \\ 0 & 0 & 2 & 6 x_{n+5 / 8} & 24 x^{2}{ }_{n+5 / 8}^{5} & 120 x^{3}{ }_{n+5 / 8}^{5} & 720 x^{4} & 5040 x^{5}+5 / 8 \\ n_{n+5} / 8\end{array}\right]\left[\begin{array}{c}a_{0} \\ a_{1} \\ a_{2} \\ a_{3} \\ a_{4} \\ a_{5} \\ a_{6} \\ a_{7}\end{array}\right]=\left[\begin{array}{c}y_{n+1 / 8} \\ y_{n+1 / 4} \\ f_{n} \\ f_{n+1 / 8} \\ f_{n+1 / 4} \\ f_{n+3 / 8} \\ f_{n+1 / 2} \\ f_{n+5 / 8}\end{array}\right]$

Finding the unknown $a_{j}, j=0(1) 7$ in equation (5) via Gaussian elimination procedures which are then filled into equation (2) to produce a continuous implicit scheme in equation (6) 
$y(t)=\alpha_{\frac{1}{8}}(t) y_{n+\frac{1}{8}}+\alpha_{\frac{1}{4}}(t) y_{n+\frac{1}{4}}+h^{2}\left[\sum_{v=0}^{\kappa} \beta_{v}(t) f_{n+v}+\beta_{0}(t) f_{n}\right]$

for $v=\frac{1}{8}, \frac{1}{4}, \frac{3}{8}, \frac{1}{2}, \frac{5}{8}$ as the hybrid points.

Now, using the transformation

$$
t=\frac{x-x_{n}}{h}, \frac{d t}{d x}=\frac{1}{h}
$$

The coefficients of $y_{n+j}$ and $f_{n+j}$ are obtained in terms of $t$ to be the continuous method for equation (6) as follows:

$$
\begin{aligned}
& \alpha_{\frac{1}{4}}=(-8 t-1) \\
& \alpha_{\frac{1}{8}}=(-8 t+2) \\
& \beta_{0}=\left[-\frac{2048}{315} t^{7}+\frac{256}{15} t^{6}-\frac{272}{15} t^{5}+10 t^{4}-\frac{137}{45} t^{3}+\frac{1}{2} t^{2}-\frac{1609}{40320} t+\frac{3}{2560}\right] \\
& \beta_{\frac{1}{8}}=\left[+\frac{2048}{63} t^{7}-\frac{3584}{45} t^{6}+\frac{1136}{15} t^{5}-\frac{308}{9} t^{4}+\frac{20}{3} t^{3}-\frac{13093}{80640} t+\frac{209}{15360}\right] \\
& \beta_{\frac{1}{4}}=\left[-\frac{4096}{63} t^{7}+\frac{6656}{45} t^{6}-\frac{1888}{15} t^{5}+\frac{428}{9} t^{4}-\frac{20}{3} t^{3}+\frac{719}{20160} t+\frac{1}{3840}\right] \\
& \beta_{\frac{3}{8}}=\left[\frac{4096}{63} t^{7}-\frac{2048}{15} t^{6}+\frac{1568}{15} t^{5}-\frac{104}{3} t^{4}+\frac{40}{9} t^{3}-\frac{247}{8064} t+\frac{7}{7680}\right] \\
& \beta_{\frac{1}{2}}=\left[-\frac{2048}{63} t^{7}+\frac{2816}{45} t^{6}-\frac{656}{15} t^{5}+\frac{122}{9} t^{4}-\frac{5}{3} t^{3}+\frac{467}{40320} t-\frac{1}{2560}\right] \\
& \beta_{\frac{5}{8}}=\left[\frac{2048}{315} t^{7}-\frac{512}{45} t^{6}+\frac{112}{15} t^{5}-\frac{20}{9} t^{4}+\frac{4}{15} t^{3}-\frac{149}{80640} t+\frac{1}{15360}\right]
\end{aligned}
$$

Evaluating the continuous method at the non-interpolation points gives the following equations

$$
y_{n+\frac{5}{8}}=4 y_{n+\frac{1}{4}}-3 y_{n+\frac{1}{8}}+\frac{h^{2}}{7680}\left[-f_{n}+127 f_{n+\frac{1}{2}}+2 f_{n+\frac{1}{4}}+32 f_{n+\frac{1}{8}}+8 f_{n+\frac{3}{8}}+8 f_{n+\frac{5}{8}}\right]
$$

(8)

$$
y_{n+\frac{3}{8}}=2 y_{n+\frac{1}{4}}-y_{n+\frac{1}{8}}+\frac{h^{2}}{15360}\left[-f_{n}-f_{n+\frac{1}{2}}+194 f_{n+\frac{1}{4}}+24 f_{n+\frac{1}{8}}+24 f_{n+\frac{3}{8}}\right]
$$

(9)

$$
y_{n+\frac{1}{2}}=3 y_{n+\frac{1}{4}}-2 y_{n+\frac{1}{8}}+\frac{h^{2}}{15360}\left[-2 f_{n}+22 f_{n+\frac{1}{2}}+412 f_{n+\frac{1}{4}}+47 f_{n+\frac{1}{8}}+242 f_{n+\frac{3}{8}}-f_{n+\frac{5}{8}}\right]
$$




$$
y_{n}=2 y_{n+\frac{1}{8}}-y_{n+\frac{1}{4}}+\frac{h^{2}}{15360}\left[18 f_{n}-6 f_{n+\frac{1}{2}}+4 f_{n+\frac{1}{4}}+209 f_{n+\frac{1}{8}}+14 f_{n+\frac{3}{8}}+f_{n+\frac{5}{8}}\right]
$$

Evaluating the first derivative of the continuous scheme at all the points yields

$$
\begin{aligned}
& y_{n}^{\prime}=-\frac{1}{80640 h}\left(645120 y_{n+\frac{1}{8}}-645120 y_{n+\frac{1}{4}}+3218 h^{2} f_{n}-934 h^{2} f_{n+\frac{1}{2}}-2876 h^{2} f_{n+\frac{1}{4}}+13093 h^{2} f_{n+\frac{1}{8}}+2470 h^{2} f_{n+\frac{3}{8}}+149 h^{2} f_{n+\frac{5}{8}}\right) \\
& y_{n+\frac{1}{8}}^{\prime}=\frac{1}{80640 h}\left(645120 y_{n+\frac{1}{4}}-645120 y_{n+\frac{1}{8}}+107 h^{2} f_{n}-277 h^{2} f_{n+\frac{1}{2}}-2710 h^{2} f_{n+\frac{1}{4}}-3104 h^{2} f_{n+\frac{1}{8}}+904 h^{2} f_{n+\frac{3}{8}}+40 h^{2} f_{n+\frac{5}{8}}\right) \\
& y_{n+\frac{1}{4}}^{\prime}=-\frac{1}{80640 h}\left(645120 y_{n+\frac{1}{8}}-645120 y_{n+\frac{1}{4}}+82 h^{2} f_{n}-262 h^{2} f_{n+\frac{1}{2}}-4444 h^{2} f_{n+\frac{1}{4}}-1355 h^{2} f_{n+\frac{1}{8}}+902 h^{2} f_{n+\frac{3}{8}}+37 h^{2} f_{n+\frac{5}{8}}\right) \\
& y_{n+\frac{3}{8}}^{\prime}=-\frac{1}{80640 h}\left(645120 y_{n+\frac{1}{8}}-645120 y_{n+\frac{1}{4}}+5 h^{2} f_{n}+389 h^{2} f_{n+\frac{1}{2}}-10058 h^{2} f_{n+\frac{1}{4}}-704 h^{2} f_{n+\frac{1}{8}}-4712 h^{2} f_{n+\frac{3}{8}}-40 h^{2} f_{n+\frac{5}{8}}\right) \\
& y_{n+\frac{1}{2}}^{\prime}=-\frac{1}{80640 h}\left(645120 y_{n+\frac{1}{8}}-645120 y_{n+\frac{1}{4}}+82 h^{2} f_{n}-4070 h^{2} f_{n+\frac{1}{2}}-8252 h^{2} f_{n+\frac{1}{4}}-1243 h^{2} f_{n+\frac{1}{8}}-11866 h^{2} f_{n+\frac{3}{8}}+149 h^{2} f_{n+\frac{5}{8}}\right) \\
& y_{n+\frac{5}{8}}^{\prime}=-\frac{1}{80640 h}\left(645120 y_{n+\frac{1}{4}}-645120 y_{n+\frac{1}{8}}+107 h^{2} f_{n}+14059 h^{2} f_{n+\frac{1}{2}}+11626 h^{2} f_{n+\frac{1}{4}}+32 h^{2} f_{n+\frac{1}{8}}+6280 h^{2} f_{n+\frac{3}{8}}+3176 h^{2} f_{n+\frac{5}{8}}\right)
\end{aligned}
$$

Equations (8) - (17) are combined in matrix form, using the matrix inversion, the methods produce a block method of the form:

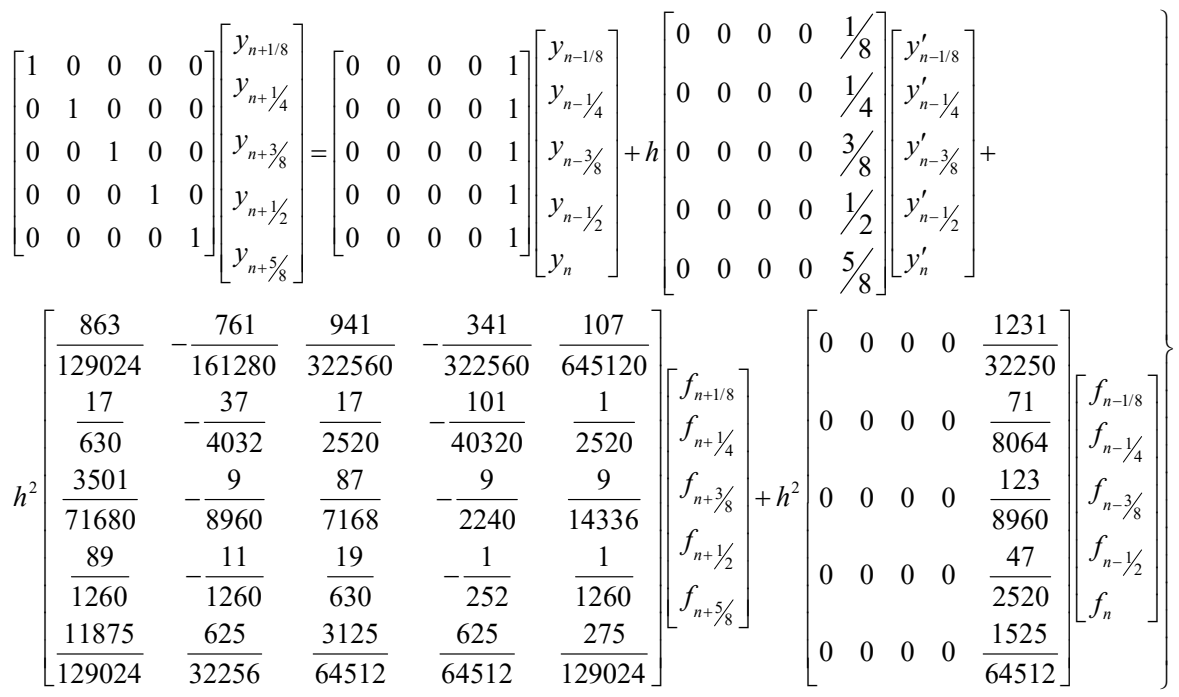


Substituting the schemes that made up the block into equations (12) to (17), gives

$$
\begin{aligned}
& y_{n+\frac{1}{2}}^{\prime}=y_{n}^{\prime}+h\left[\frac{7}{180} f_{n}+\frac{7}{180} f_{n+\frac{1}{2}}+\frac{1}{15} f_{n+\frac{1}{4}}+\frac{8}{45} f_{n+\frac{1}{8}}+\frac{8}{45} f_{n+\frac{3}{8}}\right] \\
& y_{n+\frac{1}{4}}^{\prime}=y_{n}^{\prime}+h\left[\frac{7}{180} f_{n}-\frac{1}{120} f_{n+\frac{1}{2}}+\frac{7}{360} f_{n+\frac{1}{4}}+\frac{43}{240} f_{n+\frac{1}{8}}+\frac{7}{360} f_{n+\frac{3}{8}}+\frac{1}{720} f_{n+\frac{5}{8}}\right] \\
& y_{n+\frac{1}{8}}^{\prime}=y_{n}^{\prime}+h\left[\frac{95}{2304} f_{n}-\frac{173}{11520} f_{n+\frac{1}{2}}-\frac{133}{1920} f_{n+\frac{1}{4}}+\frac{1427}{11520} f_{n+\frac{1}{8}}+\frac{241}{5760} f_{n+\frac{3}{8}}+\frac{3}{1280} f_{n+\frac{5}{8}}\right] \\
& y_{n+\frac{5}{8}}^{\prime}=y_{n}^{\prime}+h\left[\frac{95}{2304} f_{n}+\frac{125}{768} f_{n+\frac{1}{2}}+\frac{125}{1152} f_{n+\frac{1}{4}}+\frac{125}{768} f_{n+\frac{1}{8}}+\frac{125}{1152} f_{n+\frac{3}{8}}+\frac{95}{2304} f_{n+\frac{5}{8}}\right] \\
& y_{n+\frac{3}{8}}^{\prime}=y_{n}^{\prime}+h\left[\frac{51}{1280} f_{n}-\frac{21}{1280} f_{n+\frac{1}{2}}+\frac{57}{640} f_{n+\frac{1}{4}}+\frac{219}{1280} f_{n+\frac{1}{8}}+\frac{57}{640} f_{n+\frac{3}{8}}+\frac{3}{1280} f_{n+\frac{5}{8}}\right] \\
& y_{n+\frac{5}{8}}^{\prime}=y_{n}^{\prime}+h\left[\frac{95}{2304} f_{n}+\frac{125}{768} f_{n+\frac{1}{2}}+\frac{125}{1152} f_{n+\frac{1}{4}}+\frac{125}{768} f_{n+\frac{1}{8}}+\frac{125}{1152} f_{n+\frac{3}{8}}+\frac{95}{2304} f_{n+\frac{5}{8}}\right]
\end{aligned}
$$

\section{ANALYSIS OF THE BASIC PROPERTIES OF THE METHOD}

To validate the accuracy and efficiency of the block method, the basic properties of the method like order and error constant, zero stability, consistency, and its convergence were probed.

\subsection{Order and Local Truncation Error}

Adopting the method of Lambert (1973) method for finding the order of a numerical scheme also applied to equation (18). Hence, the new hybrid block method is of uniform order $\left[\begin{array}{lllll}7 & 7 & 7 & 7 & 7\end{array}\right]^{T}$ with Local

Truncation Error of $\left[\begin{array}{lllll}-\frac{33953}{362880} & \frac{41447}{762333} & \frac{2117}{32431} & -\frac{3133}{52677} & -\frac{1123}{17477}\end{array}\right]^{T}$

\subsection{Consistency}

For a linear multistep method to be consistent, the following criteria must be met.

Condition 1: $p \geq 1$

Condition 2: $\sum_{j=0}^{k} \alpha_{j}=0$ where $j=\left(\begin{array}{lll}0 & \cdots & 2\end{array}\right)$

Condition 3: $\rho^{\prime}(r)=0$ when $r=1$

Condition 4: $\rho^{\prime \prime}(r)=2 ! \sigma(r)$ when $r=1$

where $\rho$ and $\sigma$ are the first and second characteristic polynomials of (9), applying these conditions to (9), the method was found to be consistent. 


\subsection{Zero Stability of the Block}

Definition: The block is said to be zero stable if the roots $z_{s} s=1,2,3, \ldots, n$ of the characteristics polynomial $\rho(z)$ defined by $\rho(z)=\operatorname{det}(z A-E)$ satisfies $\left|z_{s}\right| \leq 1$ and the roots $\left|z_{s}\right|=1$ is simple.

For the hybrid method,

$A=\left[\left(\begin{array}{lllll}1 & 0 & 0 & 0 & 0 \\ 0 & 1 & 0 & 0 & 0 \\ 0 & 0 & 1 & 0 & 0 \\ 0 & 0 & 0 & 1 & 0 \\ 0 & 0 & 0 & 0 & 0\end{array}\right)-\left(\begin{array}{lllll}0 & 0 & 0 & 0 & 1 \\ 0 & 0 & 0 & 0 & 1 \\ 0 & 0 & 0 & 0 & 1 \\ 0 & 0 & 0 & 0 & 1 \\ 0 & 0 & 0 & 0 & 1\end{array}\right)\right]=0$

$A=z^{5}-z^{4}=0, z=0,0,0,0,1$

Hence the block is zero stable. Lambert (1973) and Ogunware and Omole (2020)

\subsection{Convergence}

Theorem 1: Convergence Lambert (1973), Fatunla (1988)

The necessary and sufficient condition for a linear multistep method to be convergent is for it to be consistent and zero stable. From the theorem above, our block method is convergent.

\section{NUMERICAL EXAMPLES}

The accuracy of the method for the direct solution of (1) is tested on some stiff second order ODE problems.

The obtained results are shown on the tables.

\section{Problem 1:}

$y^{\prime \prime}=y^{\prime}, y(0)=0, y^{\prime}(0)=-1, h=0.1$

Exact Solution: $y(x)=1-e^{x}$

\section{Problem 2:}

$y^{\prime \prime}+1001 y^{\prime}+1000 y=0$

$y(0)=1, y^{\prime}(0)=-1, h=0.05$

Exact Solution: $y(x)=e^{-x}$ 


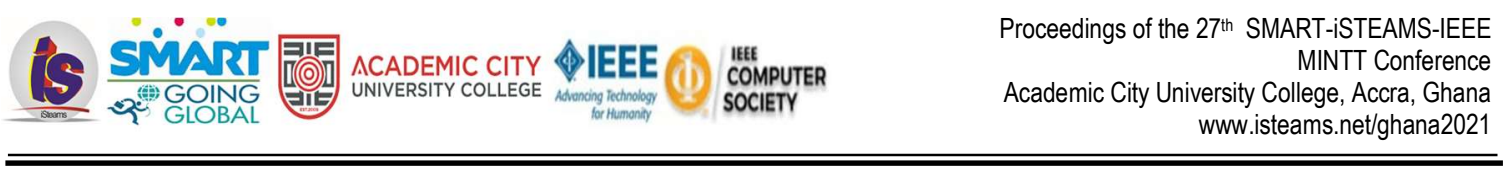

Table 1: Comparison of the result of the developed scheme with the result of Adoghe and Omole (2017) and Abada et al (2017) for Problem 1

\begin{tabular}{|l|l|l|l|l|l|}
\hline$x$ & Exact Solution & Computed Solution & Error & $\begin{array}{l}\text { Error in Adoghe } \\
\text { and Omole (2017) }\end{array}$ & $\begin{array}{l}\text { Error Abada } \\
\text { (2017) et al }\end{array}$ \\
\hline 0.1 & -0.001250781575622639 & -0.001250781575622584 & $5.52 \mathrm{e}-17$ & $1.11 \mathrm{e}-16$ & $8.00 \mathrm{e}-13$ \\
\hline 0.2 & -0.003757040047308369 & -0.003757040047308429 & $6.07 \mathrm{e}-17$ & $2.77 \mathrm{e}-16$ & $1.40 \mathrm{e}-12$ \\
\hline 0.3 & -0.006269572003761992 & -0.006269572003762011 & $1.90 \mathrm{e}-17$ & $5.55 \mathrm{e}-16$ & $3.07 \mathrm{e}-12$ \\
\hline 0.4 & -0.011313519223611346 & -0.011313519223611285 & $6.07 \mathrm{e}-17$ & $9.43 \mathrm{e}-16$ & $4.55 \mathrm{e}-12$ \\
\hline 0.5 & -0.008788393148316143 & -0.008788393148316228 & $8.50 \mathrm{e}-17$ & $9.99 \mathrm{e}-16$ & $7.43 \mathrm{e}-12$ \\
\hline 0.6 & -0.007528195444533870 & -0.007528195444533939 & $6.93 \mathrm{e}-17$ & $2.55 \mathrm{e}-15$ & $1.01 \mathrm{e}-11$ \\
\hline 0.7 & -0.010050167084167949 & -0.010050167084168019 & $6.93 \mathrm{e}-17$ & $6.88 \mathrm{e}-15$ & $1.47 \mathrm{e}-11$ \\
\hline 0.8 & -0.012578451540634417 & -0.012578451540633879 & $5.37 \mathrm{e}-16$ & $1.28 \mathrm{e}-14$ & $1.91 \mathrm{e}-11$ \\
\hline 0.9 & -0.015113064615718930 & -0.015113064615078900 & $6.40 \mathrm{e}-13$ & $1.90 \mathrm{e}-14$ & $2.59 \mathrm{e}-11$ \\
\hline 1.0 & -0.013844966011693938 & -0.013844966011373763 & $3.20 \mathrm{e}-13$ & $2.62 \mathrm{e}-14$ & $3.28 \mathrm{e}-11$ \\
\hline
\end{tabular}

Table 2: Comparison of the result of the developed scheme with the result of Abada et al (2017) and Jator (2007) for Problem 2

\begin{tabular}{|l|l|l|l|l|l|}
\hline$x$ & Exact Solution & Computed Solution & Error & $\begin{array}{l}\text { Error in Abada } \\
(2017) \text { et al }\end{array}$ & $\begin{array}{l}\text { Jator } \\
(2007)\end{array}$ \\
\hline 0.1 & 0.998438720067590380 & 0.998438720067590380 & $0.00 \mathrm{e}+00$ & $1.00 \mathrm{e}-14$ & $6.98 \mathrm{e}-12$ \\
\hline 0.2 & 0.999219055096323920 & 0.999219055096323920 & $0.00 \mathrm{e}+00$ & $3.00 \mathrm{e}-14$ & $1.00 \mathrm{e}-12$ \\
\hline 0.3 & 0.999609451284012130 & 0.999609451284012130 & $0.00 \mathrm{e}+00$ & $4.00 \mathrm{e}-14$ & $7.88 \mathrm{e}-12$ \\
\hline 0.4 & 0.998828811377365460 & 0.998828811377365340 & $1.11 \mathrm{e}-16$ & $5.00 \mathrm{e}-14$ & $1.04 \mathrm{e}-11$ \\
\hline 0.5 & 0.998048781107475520 & 0.998048781107475410 & $1.11 \mathrm{e}-16$ & $6.00 \mathrm{e}-14$ & $6.32 \mathrm{e}-11$ \\
\hline 0.6 & 0.996490547573966490 & 0.996490547573814830 & $1.51 \mathrm{e}-13$ & $7.00 \mathrm{e}-14$ & $1.00 \mathrm{e}-11$ \\
\hline 0.7 & 0.997269359998249500 & 0.997269359998244620 & $4.88 \mathrm{e}-15$ & $7.00 \mathrm{e}-14$ & $9.36 \mathrm{e}-12$ \\
\hline 0.8 & 0.997658994437520710 & 0.997658994437520490 & $2.22 \mathrm{e}-16$ & $7.00 \mathrm{e}-14$ & $2.64 \mathrm{e}-12$ \\
\hline 0.9 & 0.996879877730208140 & 0.996879877730172170 & $3.59 \mathrm{e}-14$ & $7.00 \mathrm{e}-14$ & $1.06 \mathrm{e}-11$ \\
\hline 1.0 & 0.996101369470117510 & 0.996101369469654880 & $4.62 \mathrm{e}-13$ & $7.00 \mathrm{e}-14$ & $2.32 \mathrm{e}-11$ \\
\hline
\end{tabular}

\section{DISCUSSION OF RESULTS}

The results produced by the proposed method for solving stiff second order ODEs problems 1 and 2 are displayed in table (1) and (2). Table 1 shows the results generated for problem 1 using the proposed method in comparison with the method of Adoghe and Omole (2017) and Abada (2017). The new method performs better than the methods of Adoghe and Omole (2017) and Abada (2017). It was observed that the proposed method converges faster in terms of errors. Presented in table 2 is the result produced by the developed scheme in comparison with the results of Abada (2017) and Jator (2007). In terms of errors, the result of the new method is more superior to the method of Abada (2017) and Jator (2007). 


\section{CONCLUSION}

In this research, we have derived, analyzed and implemented a new hybrid block method to directly solve stiff second-order ODEs by adopting power series as the basis function. The generated results reveal that the developed hybrid block method is more accurate than the existing method. The new method is therefore recommended for the solution of stiff second order ODEs.

\section{REFERENCES}

1. Olanegan O. O., Ogunware B. G. and Alakofa C. O. Implicit Hybrid Points Approach for solving General Second Order Ordinary Differential Equations with Initial Values. Journal of Advances in Mathematics and Computer Science 27(3): 1-14, 2018.

2. Waeleh N. and Majid Z. A. Numerical Algorithm of Block Method for General Second Order ODEs using Variable Step Size. Sains Malaysiana 46(5), 817 - 824, 2017.

3. Adoghe L. O. and Omole E. O. Comprehensive Analysis of 3-Quarter-Step Collocation Method for Direct Integration of Second order Ordinary Differential Equations Using Taylor Series Functions. Abacus (Mathematical Science Series), 44(2), 311 - 321, 2017.

4. Abada A. A., Aboiya T., \& Awari Y. S. Two-Step Block Hybrid Multistep Method for the Solution of $y^{\prime \prime}=f\left(x, y, y^{\prime}\right)$ Academic Journal of Applied Mathematical Sciences, 3(4), 40 - 45, 2017.

5. Olanegan, O. O., Awoyemi, D. O., Ogunware, B. G. and Obarhua, F. O. Continuous Double-Hybrid Point Method for the Solution of Second Order Ordinary Differential Equations. International Journal of Advanced Scientific and Technical Research 2(5), 549 - 562, 2015.

6. Awoyemi D. O., Olanegan O. O. and Akinduko, O. B. A 2-Step Four-Point Hybrid Linear Multistep Method for Solving Second Order Ordinary Differential Equations Using Taylor's Series Approach. British Journal of Mathematics \& Computer Science, 11(3): 1-13, 2015.

7. Jator S. N. A Sixth Order Linear Multistep Method for Direct Solution of $y^{\prime \prime}=f\left(x, y, y^{\prime}\right)$ International Journal of Pure and Applied Mathematics, 40, 457 - 472, 2007.

8. Awoyemi, D. O. A new sixth order algorithms for General Second order ordinary differential equation. Inter. J. Computer Math., 77, 117 - 124, 2001.

9. Omar Z. and Kuboye J. O. (2016), New seven-step numerical method for direct solution of fourth order ordinary differential equations. Journal of mathematical and fundamental sciences 48(2), 94-105.

10. Ogunware B. G. and Omole E. O (2020), A Class of Irrational Linear Multistep Block Method for the Direct Numerical Solution of Third Order Ordinary Differential Equations. Turkish Journal of Analysis and Number Theory, 2020, Vol. 8, No. 2, 21-27

11. Akeremale O. C., Kuboye, J. O, Yeak, S. H, Abununyi, E. A, Olaiju S, (2020). 'Hybrid-block numerical method for solving second order ordinary differential equations'. International Journal of Computational Analysis, 3 (2), 25 - 35.

12. Lambert, J. D. (1973): Computational methods in ordinary differential equation, John Wiley \& Sons Inc. New York.

13. Fatunla, S. O. (1988): Numerical methods for initial value problems in ordinary differential equations, Academic press inc. Harcourt Brace Jovanovich Publishers, New York. 